\title{
All-fiber highly-chirped dissipative soliton generation in the telecom range
}

\author{
Denis S. Kharenko ${ }^{1,2,}{ }^{*}$, Innokentiy S. Zhdanov ${ }^{1,2}$, AnAstasia E. Bednyakova 2,3 , Evgeniy V. \\ Podivilov $^{1,2}$, Mikhail P. Fedoruk ${ }^{2,3}$, Alexander Apolonski ${ }^{1,4}$, Sergei K. Turitsyn ${ }^{5,2}$, AND \\ SERGEY A. BABIN ${ }^{1,2}$ \\ ${ }^{1}$ Institute of Automation and Electrometry, SB RAS, Novosibirsk 630090, Russia \\ ${ }^{2}$ Novosibirsk State University, Novosibirsk 630090, Russia \\ ${ }^{3}$ Institute of Computational Technologies SB RAS, Novosibirsk 630090, Russia \\ ${ }^{4}$ Ludwig-Maximilians-Universitaet and Max-Planck-Institut fuer Quantenoptik, Garching D-85748, Germany \\ ${ }^{5}$ Aston Institute of Photonic Technologies, Aston University, Birmingham B4 7ET, UK \\ *Corresponding author: kharenko@iae.nsk.su \\ Compiled July 19, 2017
}

\begin{abstract}
A high-energy (0.93 nJ) all-fiber Erbium femtosecond oscillator operating in the telecom spectral range is proposed and realized. The laser cavity built of commercially available fibers and components combines non-PM and PM parts providing stable generation of highly-chirped (chirp parameter 40) pulses compressed in an output piece of standard PM fiber to $165 \mathrm{fs}$. The results of numerical simulation agree well with experiment. The analyzed intracavity pulse dynamics enables the classification of the generated pulses as dissipative solitons. (C) 2017 Optical Society of America
\end{abstract}

OCIS codes: (140.3510) Lasers, fiber; (140.3500) Lasers, erbium; (140.4050) Mode-locked lasers; (140.7090) Ultrafast lasers; (060.5530) Pulse propagation and temporal solitons.

http://dx.doi.org/10.1364/ao.XX.XXXXXX

Recent progress in the development of high-power femtosecond fiber lasers operating around $1 \mu \mathrm{m}$, based on the highlychirped dissipative solitons (HCDS) generated in all-normal dispersion (ANDi) laser cavities [1-3] paved the way for the transfer and expansion of this technology into other spectral regions. The first natural candidate would be the telecom window $(\sim 1.5 \mathrm{mi}-$ cron), where high power mode-locked fiber lasers have a wide range of practical applications, from CARS spectroscopy [4] to few-cycle pulse synthesis [5], terahertz-wave generation [6], frequency metrology [7, 8] and, of course, telecommunications [9]. The advantage of this spectral interval is the availability of various low cost telecom components that might make such lasers commercially attractive.

For the most of practical applications, long-term stability and reliability as well as output power, pulse duration and output beam quality are five important laser characteristics. From this point of view, all-fiber lasers have advantages due to their compactness, relative simplicity of the schemes, high efficiency and perfect beam quality. There is though one quite important weakness - a polarization state that is undefined in standard single-mode fibers (SMFs) leading to uncontrollable variation with the changing environment, especially in a long enough fiber cavity. Polarization maintaining (PM) fibers are widely used to mitigate this problem. As a result, $150 \mathrm{fs}, 25 \mathrm{pJ}$ pulses were demonstrated recently in an all-fiber all-PM Erbium laser with graphene saturable absorber (GSA) operated in the anomalous net dispersion regime [10]. Slightly higher energy (44 pJ) for 174 fs pulses was demonstrated previously in non-PM cavity with GSA [11]. Another type of saturable absorber, topological insulator (TI) was also successfully demonstrated in an all-fiber configuration resulting in 130-fs 45-pJ pulses [12].

Further energy up-scaling became possible by realizing the HCDS in the normal net dispersion regime, one of the most advanced ways to generate high-energy femtosecond pulses in mode-locked oscillators demonstrated so far, see review [13]. We have studied the HCDS regime earlier [14], showing an essential result used in the current publication, namely that the numerical simulations and analytical solution in the high chirp limit $(f \gg 1)$ agree well at $f>10$. The HCDS regime was experimentally demonstrated for the first time in Ytterbium [15] and Erbium fiber oscillators [16]. All the following results cited in this short literature review will be devoted to Erbium oscillators. For the TI mode-locked oscillators, $170 \mathrm{fs}, 0.21 \mathrm{~nJ}$ pulses were demonstrated [17] showing thus 5-fold pulse energy increase at similar pulse durations in comparison to the results reported previously for the anomalous dispersion regime [12]. Oscillators mode-locked with a semiconductor saturable absorber mirror (SESAM), another conventional technique [18-20] provided significantly longer, 528 fs pulses of $1.8 \mathrm{~nJ}$ energy [18], or $700 \mathrm{fs}, 38 \mathrm{~nJ}$ [20]. This approach breaks an all-fiber concept and requires several intracavity bulk components. The use of a single-wall carbon nanotube saturable absorber (SWCNT-SA) demonstrated high scalability from $6.5 \mathrm{~nJ}$ (1 ps duration) [21] up to $34 \mathrm{~nJ}$ (370 fs pulse duration) [22] in spite of non-PM cavity design. An all-fiber cavity approach with nonlinear polarization evolution (NPE), another type of a mode-locking mechanism, resulted in $111 \mathrm{fs}, 0.2 \mathrm{~nJ}$ pulses [23]. This combination of the pulse parameters represents the shortest duration and the high- 


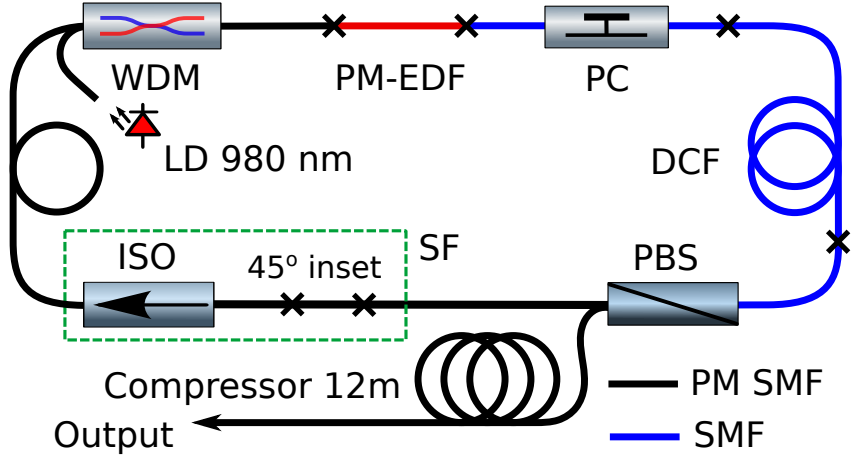

Fig. 1. Experimental setup of an all-fiber Erbium HCDS oscillator.

est energy pulses generated so far in all-fiber Erbium oscillators, at least in publications containing necessary pulse characterisation (interferometric autocorrelation traces, radio-frequency (RF) beat signal etc.). Schemes with NPE mode-locking realized with bulk optics elements provide much better combination of the pulse parameters [24-26], specifically 10 nJ, 75 fs.

Note also that 1480-nm pump gives additional benefit in comparison to that at $980 \mathrm{~nm}$. For instance, the pulse energy generated with 980-nm laser diodes (LDs) [25] is three times lower than that reported with 1480-nm LDs in a similar scheme [26], $3.5 \mathrm{~nJ}$ and $10 \mathrm{~nJ}$ respectively.

In this work we demonstrate an all-fiber Erbium oscillator providing stable generation of highly-chirped (chirp parameter $f \sim 40$ ) dissipative solitons (HCDSs). Here we successfully apply the approach previously developed for an Ytterbium allfiber laser [27] to an Erbium fiber laser. To provide stable NPE mode-locking we combined the laser cavity from non-PM and PM parts and optimized the corresponding lengths for the best performance. In order to achieve the generation of HCDSs, several conditions have to be satisfied: normal net dispersion of the laser cavity, strong spectral filtering and sufficiently high signal amplification providing nonlinear pulse build-up. Our design is based on commercially available dispersion compensating (DCF) and PM optical fibers and components.

Fig. 1 illustrates the used oscillator scheme that is similar to the ring scheme of the NPE mode-locked Ytterbium fiber oscillator presented in [27]. The all-fiber cavity comprises a 6-mlong DCF with large normal dispersion $\left(48 \mathrm{ps}^{2} / \mathrm{km}\right.$ at $1550 \mathrm{~nm}$, DCF-38, Thorlabs Inc.) and a 5-m-long PM fiber with typical anomalous dispersion $\left(-23 \mathrm{ps}^{2} / \mathrm{km}\right)$ that consists of both the fiber components with their fiber tails and the active fiber. An additional $0.5 \mathrm{~m}$ piece of standard SMF is a part of the polarization beam splitter (PBS) tail. NPE rejection port was used as an oscillator output and defined the output polarization extinction ratio at $20-\mathrm{dB}$ level. The net cavity dispersion is normal and was estimated as $0.165 \mathrm{ps}^{2}$. For signal amplification, a $1.3 \mathrm{~m}$-long PM Erbium-doped fiber with anomalous dispersion of about $-20 \mathrm{ps}^{2} / \mathrm{km}$ was used (Nufern, PM-ESF-7/125). Spectral filtering (SF) was provided with an all-fiber polarization-based Lyot filter with sinusoidal transmission function. It was implemented with a 250-mm-long PM inset spliced at an angle of 45 degrees to the main cavity axis and a polarization sensitive isolator (ISO).

The output pulses were compressed in a 12-m-long standard PM fiber (Nufern, PM1550-XP). Our all-fiber laser provides stable mode-locking via NPE in the DCF part which is self-starting

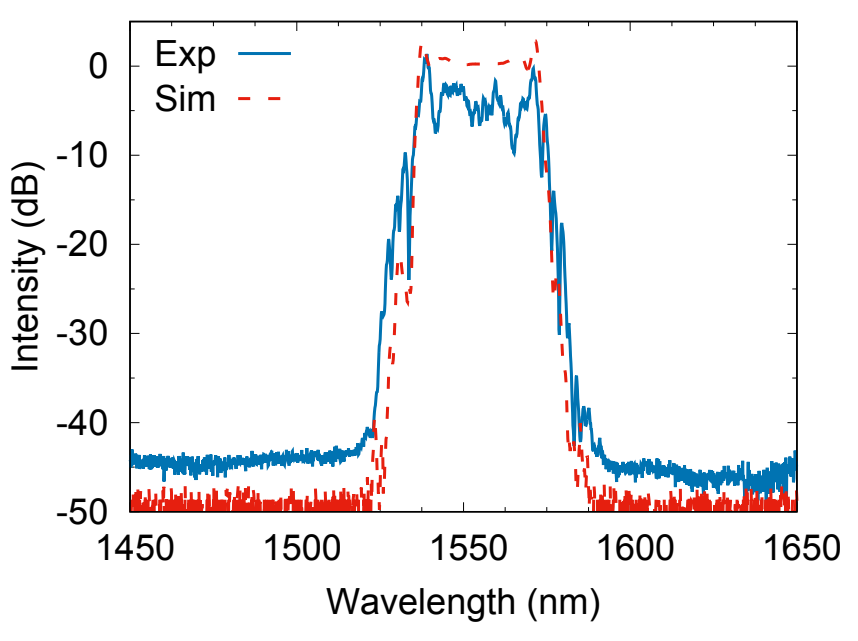

Fig. 2. Output spectra in the experiment and simulation.

at an appropriate polarization controller (PC) adjustment. The output pulse energy can be increased at higher pump power accompanied by PC readjustment, resulting in a self-sustaining regime of operation. The optical spectrum of the generated pulses was characterized by a conventional optical spectrum analyzer (Yokogawa 6370). We also used an autocorrelator (Avesta Ltd. AA-20DD) with a 30 ps time delay range to measure the duration of the chirped and compressed pulses. As we exploited the co-propagating pump scheme, a part of the residual pump could pass to the laser output. To avoid its impact on ACF and power measurements, a dichroic mirror was applied.

To achieve maximum output energy and spectral bandwidth simultaneously, we varied the bandwidth of SF and the lengths of DCF and PM fibers. We found that the cavity with longer DCF section needs a narrower SF bandwidth in order to form stable DS pulses. Note, that there is a limit on the DCF length in this scheme. A further increase of DCF length (beyond 9 meters) leads to a NPE overdriving and reduced mode-locking stability.

In order to optimize the regime, we also performed numerical simulations based on the generalized nonlinear Schrödinger equation (NLSE), with the Raman scattering term. This model was developed previously and described in detail in [28]. The fiber laser scheme used in the simulation comprises a 6-mlong DCF with the following parameters: second-order dispersion coefficient $\beta_{2}=48 \mathrm{ps}^{2} / \mathrm{km}$, third-order dispersion $\beta_{3}=-0.25$ $\mathrm{ps}^{3} / \mathrm{km}$ and nonlinearity coefficient $\gamma=3 \mathrm{~W}^{-1} \mathrm{~km}^{-1}$ and 5.4$\mathrm{m}$-long PM fiber $\left(\beta_{2}=-23 \mathrm{ps}^{2} / \mathrm{km}, \beta_{3}=0.037 \mathrm{ps}^{3} / \mathrm{km}\right.$ and $\gamma=2$ $\mathrm{W}^{-1} \mathrm{~km}^{-1}$ ). The effects of mode-locking, gain and filtering are treated as point-action. The spectral filtering is implemented as a multiplication of the optical field and the filter transmission spectrum in the frequency domain. The transmission spectrum of the Lyot filter is approximated by sinusoidal function with the period and maximum reflectivity corresponding to the experiment. The gain spectrum used in the simulation corresponds to the experimentally measured one with total small signal gain $g_{0}$ equal to $30 \mathrm{~dB}$. The gain saturation is modelled according to [28].

The calculated spectrum agrees well with the experimental one at the optimal parameters (Fig. 2). It has a shape with sharp edges typical for the HCDS regime [13, 29]. At the laser output the DS has a duration of $\sim 6$ ps and reaches $165 \mathrm{fs}$ after dechirping. The spectrally limited duration estimated by the Fourier transformation of the output spectrum (Fig. 2) is $150 \mathrm{fs.}$ 


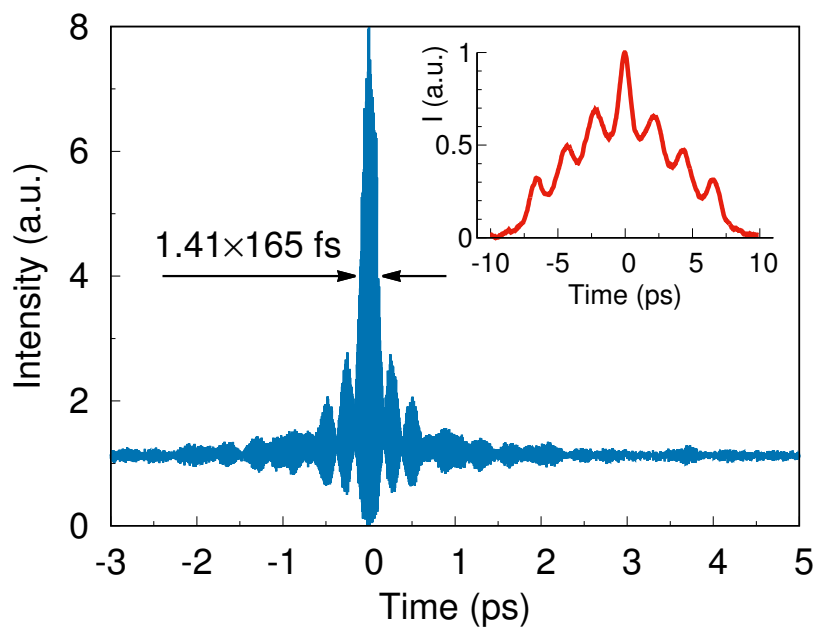

Fig. 3. Autocorrelation traces of the dechirped and chirped (inset) pulse.

So, the pulses were compressed by a factor of about 40 . This value can also be used as an estimation of the chirp parameter $[14,29]$. Fig. 3 depicts the results of the interferometric autocorrelation measurements of the compressed and original (right inset) pulses. The energy of the optimal pulses was estimated to be $0.93 \mathrm{~nJ}$ according to the average output power reaching $16 \mathrm{~mW}$ at $17.2 \mathrm{MHz}$ repetition rate. The pulse train stability was characterized by RF beat signal measurements (see. Fig. 4) that was performed with different bandwidths and spectral ranges demonstrating the high contrast level exceeding $70 \mathrm{~dB}$. Some residual timing jitter can be seen.

For a better understanding of intracavity pulse evolution we studied numerically spectral and temporal pulse variations along the cavity, depicted in Fig. 5(a). The wide range temporal and spectral profiles in logarithmic scales (see corresponding insets) show the absence of satellite pulses in numerical window and verify the obtained state as a stable. A chirped pulse with a duration of $10 \mathrm{ps}$ and a bandwidth of $38 \mathrm{~nm}$ enters PM fiber $(\mathrm{z}=0)$, where it experiences weak temporal compression due to the anomalous dispersion of the fiber, while its spectral width remains almost the same. The spectral and temporal pulse shapes corresponding to the exit point of the PM fiber (point "1") are shown in Fig. 5(b,c). Then the pulse passes through the Lyot filter, which cuts off both spectral bandwidth and temporal wings of the pulse (due to its chirped nature) resulting in its shortening from 8 to 4 ps. After strong linear filtering, the spectral width reaches its minimum value equal to $29 \mathrm{~nm}$. The pulse shape also changes significantly (point "2" in Fig. 5(b,c)). Then the pulse continues to propagate in the PM fiber. It evolves self-similarly without significant variation of spectral and temporal shape (compare points "2" and "3" in Fig. 5(b,c)). After amplification by several times in a short piece of Er-doped fiber (point " 3 ") the pulse enters DCF with large normal dispersion. At the entrance of DCF the pulse duration reaches its minimum value along the cavity (equal to $3 \mathrm{ps}$ ). During the propagation in DCF, the pulse acquires a linear chirp, resulting in a H-type (or "Batman"-type) spectral shape typical for a highly-chirped DS regime (point "4" in Fig. 5(b)). Its bandwidth gradually increases from 30 to $38 \mathrm{~nm}$ and the pulse duration shows a threefold increase (point "4" in Fig. 5(c)). Finally, after spatial separation of the orthogonal polarization components by PBS providing amplitude modulation,

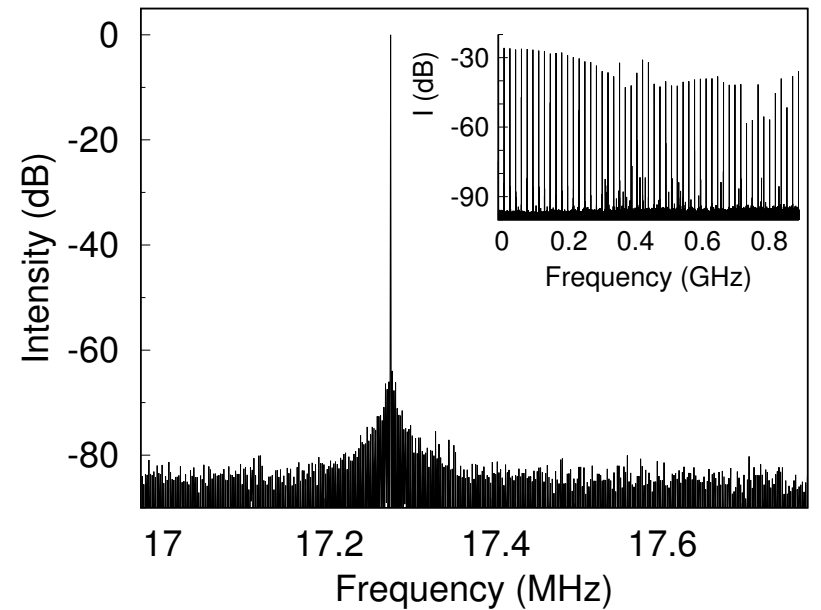

Fig. 4. RF spectrum measured with $1 \mathrm{MHz}$ span and $100 \mathrm{~Hz}$ bandwidth. Inset: the same with $1 \mathrm{GHz}$ span and $2 \mathrm{kHz}$ bandwidth.

the main component is sent to $\operatorname{PMF}(\mathrm{z}=0)$ and a new round trip starts. The remaining part goes to the output (red dashed line in Fig. 2). Its energy amounts to $2.5 \mathrm{~nJ}$ that is 2.5 times higher than the experimental value.

The pulse evolution in the laser cavity is self-consistent due to the balanced action of nonlinearity and dispersion, loss and gain [13]. The pulse reshaping (spectral filtering) is provided via losses induced by the Lyot filter. The amplitude modulation is provided by the joint action of NPE and PBS, whereas the similariton formation [30] takes place in DCF. Therefore, we can conclude that the laser realized in our experiment can be referred to as a passive self-similar oscillator, in which pulseshaping is based on both spectral filtering of a chirped pulse and self-similar evolution. The observed passive self-similar evolution is closer to the dissipative soliton regime than to the active self-similar regime with local nonlinear attraction [30], as variations of the spectral width and pulse duration along the cavity are below $50 \%$ relative to their average values. We can also support this finding in terms of nonlinear and dispersion phase shift that must be equal to each other for a highly-chirped DS [29]. As the cavity parameters change significantly along the cavity, the balance of these phase shifts should be rewritten in the integral form:

$$
\int_{0}^{L} \frac{\beta_{2}(l)}{2} \Delta(l)^{2} d l=\int_{0}^{L} \gamma(l) P_{0}(l) d l,
$$

where: $\Delta=(\delta \lambda / \lambda) \omega\left[\mathrm{ps}^{-1}\right]$ is the half width of the spectrum, with $\delta \lambda$ measured in the experiment, $P_{0}$ is a pulse peak power. In accordance with Fig. 5(a), we can identify three regions where parameters change slightly or linearly and their averaged values can be easily calculated. As the PBS coupling efficiency depends on both the PC adjustment and the regime used (continuouswave or mode-locked), we estimated this parameter equal to $50 \%$ based on Fig. 5(c) (transition from point 4 to 1 at approximately constant pulse duration). The intracavity map of the peak power could be retrieved from the output peak power equal to $0.93 \mathrm{~nJ} / 6 \mathrm{ps} \sim 150 \mathrm{~W}$. Taking into account all of the above, we estimated the nonlinear and dispersion phase shifts ratio as 0.8 , quite close to 1 . This estimation and the parameter $f \sim 40$ give us a reason to classify the generated pulses as HCDS. 

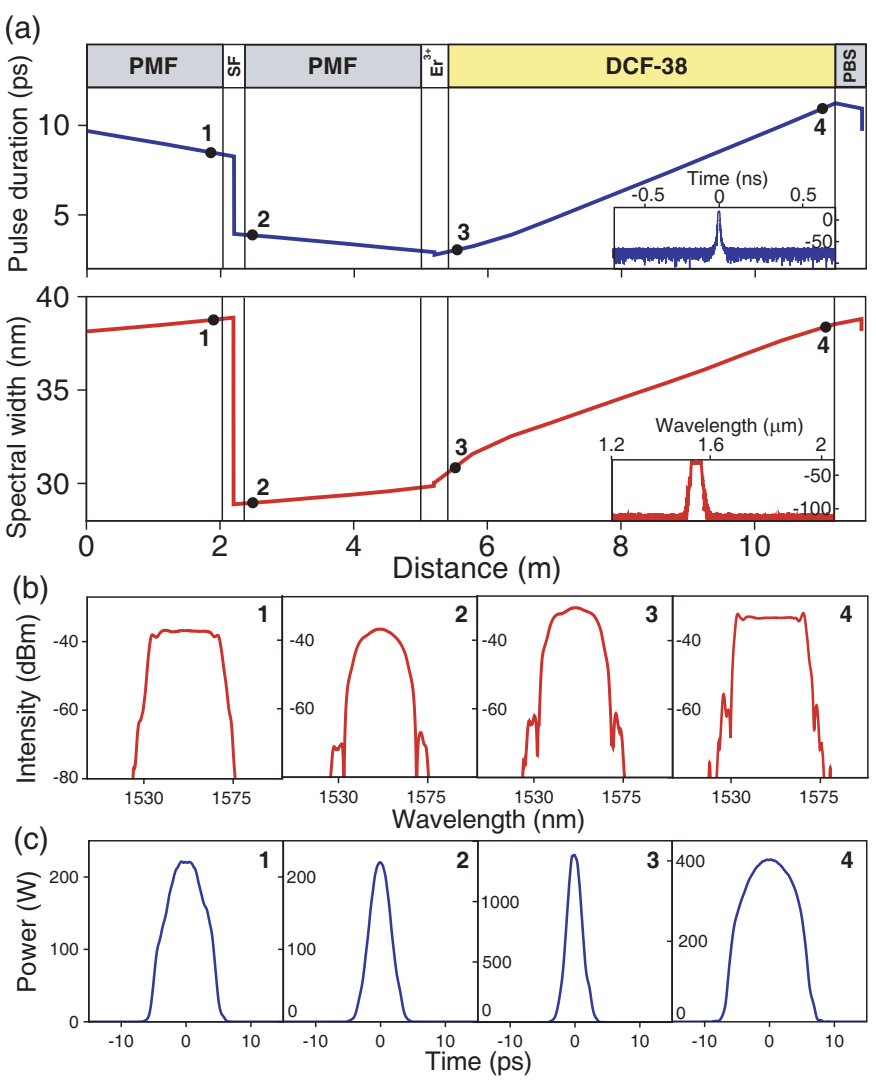

Fig. 5. (a) Variations of the pulse duration and spectral width (at -10 dB level) along the cavity with the wide range logarithmic scale temporal and spectral profiles in insets. (b-c) The corresponding spectral and temporal shapes at the cavity points $1-4$.

Note that the optical spectrum can be significantly unbalanced by detrimental effects of the third-order dispersion. However, this action can be neglected if the corresponding phase shift is much smaller than the phase shift induced by the secondorder dispersion [31] that occurs if the following condition for the signal bandwidth is satisfied:

$$
\int_{0}^{L} \frac{\beta_{3}(l)}{3} \Delta(l)^{3} d l \ll \int_{0}^{L} \frac{\beta_{2}(l)}{2} \Delta(l)^{2} d l
$$

where $\beta_{3} \approx-0.1195 \mathrm{ps}^{3} / \mathrm{km}$ is the third-order dispersion coefficient averaged over the cavity. Our estimations and the symmetric experimental spectral shape presented in Fig. 2 show that the net third-order cavity dispersion is negligible.

In conclusion, we have realized and studied an all-fiber Erbium oscillator in the telecom spectral range that combines nonPM and PM cavity parts and provides stable generation of highly chirped (chirp parameter $f \sim 40$ ) pulses compressed externally in a 12-m-long piece of standard PM fiber to $165 \mathrm{fs}$. $0.93 \mathrm{~nJ}$ pulses have been generated at $17.2 \mathrm{MHz}$ repetition rate in the cavity built of commercially available fibers and components. The energy level achieved is approximately 5 times higher than that demonstrated earlier for $\sim 100$ fs pulses generated in an all-fiber configuration $[17,23]$. Further energy upscaling seems feasible by using specialty PM fibers with a close to zero or normal dispersion value. The numerical simulation qualitatively agrees with experiment and reveals the intracavity dynamics affording a classification of the generated pulse as HCDS.

\section{FUNDING INFORMATION}

This work is supported by the Russian Foundation for Basic Research, Russian Science Foundation (grant 14-22-00118, work of DSK, ISZ, EVP and SAB, grant 17-71-20082, work of AEB) and integration project of Siberian Branch of RAS. The work of SKT was supported by the Grant of Ministry of Education and Science of the Russian Federation (agreement No. 14.B25.31.0003) and the EPSRC project UNLOC.

\section{REFERENCES}

1. A. Chong, J. R. Buckley, W. H. Renninger, and F. W. Wise, Opt. Express 14, 10095 (2006).

2. S. Lefrançois, K. Kieu, Y. Deng, J. D. Kafka, and F. W. Wise, Opt. Lett. 35, 1569 (2010).

3. D. S. Kharenko, V. A. Gonta, and S. A. Babin, Laser Phys. Lett. 13, 025107 (2016).

4. M. Marangoni, A. Gambetta, C. Manzoni, V. Kumar, R. Ramponi, and G. Cerullo, Opt. Lett. 34, 3262 (2009).

5. G. Krauss, S. Lohss, T. Hanke, A. Sell, S. Eggert, R. Huber, and A. Leitenstorfer, Nat. Photonics 4, 33 (2009).

6. A. Schneider, M. Stillhart, and P. Günter, Opt. Express 14, 5376 (2006).

7. N. R. Newbury and W. C. Swann, J. Opt. Soc. Am. B 24, 1756 (2007).

8. V. S. Pivtsov, B. N. Nyushkov, I. I. Korel, N. A. Koliada, S. A. Farnosov, and V. I. Denisov, Quantum Electron. 44, 507 (2014).

9. J. Kim and Y. Song, Adv. Opt. Photonics 8, 465 (2016).

10. J. Tarka, J. Boguslawski, G. Sobon, I. Pasternak, A. Przewloka, W. Strupinski, J. Sotor, and K. M. Abramski, IEEE J. Sel. Top. Quantum Electron. 23, 1 (2017).

11. D. Popa, Z. Sun, F. Torrisi, T. Hasan, F. Wang, and A. C. Ferrari, Appl. Phys. Lett. 97, 16 (2010).

12. J. Sotor, G. Sobon, and K. M. Abramski, Opt. Express 22, 13244 (2014).

13. P. Grelu and N. N. Akhmediev, Nat. Photonics 6, 84 (2012).

14. D. S. Kharenko, O. V. Shtyrina, I. A. Yarutkina, E. V. Podivilov, M. P. Fedoruk, and S. A. Babin, J. Opt. Soc. Am. B 28, 2314 (2011).

15. H. Lim, F. O. Ilday, and F. W. Wise, Opt. Lett. 28, 660 (2003).

16. L. M. Zhao, D. Y. Tang, and J. Wu, Opt. Lett. 31, 1788 (2006).

17. J. Boguslawski, G. Sobon, R. Zybala, and J. Sotor, Opt. Lett. 40, 2786 (2015).

18. A. Cabasse, G. Martel, and J. L. Oudar, Opt. Express 17, 9537 (2009).

19. A. Cabasse, D. Gaponov, K. Ndao, A. Khadour, J.-L. Oudar, and G. Martel, Opt. Lett. 36, 2620 (2011).

20. M. Tang, H. Wang, R. Becheker, J.-L. Oudar, D. Gaponov, T. Godin, and A. Hideur, Opt. Lett. 40, 1414 (2015).

21. Y. W. Song, S. Yamashita, and S. Maruyama, Appl. Phys. Lett. 92, 1 (2008).

22. H. Jeong, S. Y. Choi, F. Rotermund, Y.-H. Cha, D.-Y. Jeong, and D.-I. Yeom, Opt. Express 22, 22667 (2014).

23. M. Nikodem and K. M. Abramski, Opt. Commun. 283, 109 (2010).

24. N. B. Chichkov, K. Hausmann, D. Wandt, U. Morgner, J. Neumann, and D. Kracht, Opt. Lett. 35, 3081 (2010).

25. H. Liu, Z. Liu, E. S. Lamb, and F. W. Wise, Opt. Lett. 39, 1019 (2014).

26. A. Ruehl, V. Kuhn, D. Wandt, and D. Kracht, Opt. Express 16, 3130 (2008).

27. D. S. Kharenko, E. V. Podivilov, A. A. Apolonski, and S. A. Babin, Opt. Lett. 37, 4104 (2012).

28. A. E. Bednyakova, S. A. Babin, D. S. Kharenko, E. V. Podivilov, M. P. Fedoruk, V. L. Kalashnikov, and A. Apolonski, Opt. Express 21, 20556 (2013).

29. E. V. Podivilov and V. L. Kalashnikov, JETP Lett. 82, 467 (2005).

30. A. Chong, L. G. Wright, and F. W. Wise, Reports Prog. Phys. 78, 113901 (2015).

31. G. P. Agrawal, Nonlinear Fiber Optics (Academic Press, 2001), 3rd ed. 